\title{
Social Influence and Uptake of Couples HIV Testing and Counselling in KwaZulu-Natal, South Africa
}

\author{
Matthew J. Johnson ${ }^{1,2}$ (D) Lynae A. Darbes ${ }^{3,4} \cdot$ Victoria Hosegood $^{5,6} \cdot$ Mallory O. Johnson $^{3} \cdot$ Katherine Fritz $^{7}$. \\ Thulani Ngubane ${ }^{8} \cdot$ Heidi van Rooyen ${ }^{8,9} \cdot$ Nuala McGrath ${ }^{5,6,10}$
}

Accepted: 10 August 2021 / Published online: 21 August 2021

(C) The Author(s) 2021

\begin{abstract}
Social influences may create a barrier to couples HIV testing and counselling (CHTC) uptake in sub-Saharan Africa. This secondary analysis of data collected in the 'Uthando Lwethu' randomised controlled trial used discrete-time survival models to evaluate the association between within-couple average 'peer support' score and uptake of CHTC by the end of nine months' follow-up. Peer support was conceptualised by self-rated strength of agreement with two statements describing friendships outside of the primary partnership. Eighty-eight couples (26.9\%) took up CHTC. Results tended towards a dichotomous trend in models adjusted only for trial arm, with uptake significantly less likely amongst couples in the higher of four peer support score categories (OR 0.34, 95\% CI 0.18, 0.68 [7-10 points]; OR 0.53, 95\% CI 0.28, 0.99 [ $\geq 11$ points]). A similar trend remained in the final multivariable model, but was no longer significant (AOR 0.59, 95\% CI 0.25, 1.42 [7-10 points]; AOR $0.88,95 \%$ CI $0.36,2.10$ [ $\geq 11$ points]). Accounting for social influences in the design of couples-focused interventions may increase their success.
\end{abstract}

Keywords HIV prevention · Couples HIV testing and counselling $\cdot$ Social influence $\cdot$ Peer support $\cdot$ South Africa

Matthew J. Johnson

mj2@mrc.soton.ac.uk

Lynae A. Darbes

lynaed@med.umich.edu

Victoria Hosegood

v.hosegood@soton.ac.uk

Mallory O. Johnson

mallory.johnson@ucsf.edu

Katherine Fritz

kfritz@icrw.org

Thulani Ngubane

tngubane@hsrc.ac.za

Heidi van Rooyen

hvanrooyen@hsrc.ac.za

Nuala McGrath

n.mcgrath@soton.ac.uk

1 MRC Lifecourse Epidemiology Unit, Southampton General Hospital, University of Southampton, Mailpoint 95, Tremona Road, Southampton SO16 6YD, UK

2 NIHR ARC Wessex Data Science Hub, Faculty of Environmental \& Life Sciences, University of Southampton, Southampton, UK
3 Center for AIDS Prevention Studies, Division of Prevention Sciences, School of Medicine, University of California, San Francisco, San Francisco, CA, USA

4 Department of Health Behavior and Biological Sciences, Center for Sexuality and Health Disparities, University of Michigan School of Nursing, Ann Arbor, USA

5 Department of Social Statistics and Demography, Faculty of Social, Human and Mathematical Sciences, University of Southampton, Southampton, UK

6 Africa Health Research Institute, KwaZulu-Natal, South Africa

7 International Center for Research on Women, Washington, DC, USA

8 Human Sciences Research Council, Durban, South Africa

9 School of Clinical Medicine, Faculty of Health Sciences, University of the Witwatersrand, Johannesburg, South Africa

10 School of Primary Care, Population Sciences and Medical Education, Faculty of Medicine, University of Southampton, Southampton, UK 


\section{Abbreviations}

$\begin{array}{ll}\text { HIV } & \text { Human immunodeficiency virus } \\ \text { SSA } & \text { Sub-Saharan Africa } \\ \text { CHTC } & \text { Couples HIV testing and counselling } \\ \text { PS } & \text { Peer support } \\ \text { OR } & \text { Odds ratio } \\ \text { AOR } & \text { Adjusted odds ratio }\end{array}$

\section{Introduction}

Most HIV transmission in sub-Saharan Africa (SSA) occurs within marital or cohabiting couple relationships [1, 2]. Within-couple serodiscordancy is common [3, 4], but the risk of HIV transmission could still be reduced by adopting appropriate preventive behaviours [5, 6]. Knowledge of each partner's HIV status could prompt couples to reevaluate, and perhaps change, their behaviour [7], and has been associated with a decline in unprotected sex [8], particularly where serodiscordancy is known [9]. However, this knowledge has often been lacking in SSA [10-12], whether product of disinclination to seek or accept testing [13], a preference for inferring personal status from that of the partner [14, 15], or active choice not to disclose [16, 17]. Irrespective of the reason, as non-awareness of personal and/ or partner HIV status is a barrier to engagement with prevention behaviours, there is an ongoing need to expand HIV testing and facilitate mutual disclosure amongst couples.

Historically, many models of HIV prevention have prioritised the internal beliefs and motivations of the individual as determining their propensity to adopt health behaviours, but this perspective may be unable to explain actions guided by thoughts and feelings about the partner, or unequal relationship power [18]. Indeed, the inherently dyadic dimension to HIV transmission has prompted calls for greater utilisation of couples-focused interventions that explicitly recognise, address and leverage characteristics of the relationship [18, 19], with such interventions consistently shown to encourage testing and other prevention behaviours more effectively than those targeted to individuals [20, 21]. Couples HIV testing and counselling (CHTC) has been shown to precipitate a sustained reduction in unprotected sex and partner concurrency [22], and is recommended by the World Health Organisation for its emphasis on mutual testing and disclosure, followed by the delivery of tailored counselling messages to address couple status and facilitate joint decision-making [23]. Nonetheless, uptake remains low [24], suggesting further barriers for couples who might otherwise seek or accept the intervention.

According to Lewis and colleagues' interdependence model [25], couple-level behaviour change is shaped by partners' ability to transition from a purely personal approach to health risks to one that assigns them greater meaning via direct association with the relationship, and their desire to see it continue. When partners are united in their assessment of risk and the approach to its management, they are more able to make decisions and act collaboratively. Nonetheless, the primary partnership remains just one of many interpersonal relationships experienced by an individual, each of which may shape their behaviour to some extent. Personal decision-making guided by anticipation of social reward or censure, or self-comparison with others, may lead some to align themselves with norms established and maintained by their structural social context [26]. In SSA, there is evidence to suggest that social influences may discourage testing and undermine HIV prevention efforts. Fear of stigmatisation, lost personal relationships or diminished social standing following a positive result or association with HIV services may result in testing delay or avoidance [13]. Aspiration to an idealised 'masculine' self-identity framed around presenting personal qualities of self-confidence, strength and invulnerability shapes normative male attitudes to health-seeking and risk behaviours in SSA [27, 28]. Male peer groups, in particular, may perpetuate harmful normative attitudes and behaviours amongst members, by causing some to avoid association with health services for fear of projecting an image of weakness $[27,28]$, or by providing social approval, encouragement and practical advice to support concurrent [29] or extramarital relationships [30, 31].

Much as directly addressing the relationship between partners has proven an effective HIV prevention strategy, other kinds of established social relationships have emerged as a viable platform for intervention in SSA. For example, invitations to CHTC were found to have greater chance of success when issued by family members or other social acquaintances rather than hitherto unknown agents [32, 33]. Furthermore, peer leader interventions have been associated with an increase in safer sex behaviours and improved knowledge and attitudes regarding HIV risk and transmission among urban working women [34], and with increased HIV testing and reduced inequitable gender norm attitudes among male peer networks [35]. Finally, follow-up after a peer group intervention revealed a broader increase in recent testing and improvement in attitudes to condom use across the study locality [36], suggesting that new ideas, information or behaviours perceived as valuable or beneficial may have further appeal to, and be adopted by, those not directly involved in the initial intervention.

Although recent qualitative research from Kenya has suggested that social influences can reinforce gender norms and inequalities within marriage, with negative implications for HIV transmission risk [37], most studies have examined their effect upon individuals only. To further explore their effect on couples, this secondary analysis of existing data collected as part of the Uthando Lwethu ('Our Love') randomised controlled trial evaluated the association between 
men's, women's and couples' perceived access to peer support and the outcome of CHTC uptake, in the context of other demographic and relationship factors.

\section{Methods}

\section{Primary Study}

The Uthando Lwethu trial was an efficacious behavioural couples-focused intervention that significantly increased CHTC uptake in rural KwaZulu-Natal province, South Africa, under the hypothesis that couples with improved relationship quality following couples counselling would be more likely to take up CHTC together [38]. Heterosexual couples aged 18 to 50 years in a sexually active relationship of minimum six months' duration were recruited from the Vulindlela sub-district of KwaZulu-Natal. Those with prior mutual disclosure or history of couples testing were excluded. Recruitment took place from March 2012 to August 2014, at which time the 15-49 age group accounted for more than half of the provincial population [39] and had HIV prevalence at $27.9 \%$ [40]. Overall, 332 couples were recruited and followed for nine months, during which time six broke up and sixteen were lost to follow-up. Immediately following randomisation all intervention and control couples were eligible for CHTC provided by study staff, and received text message reminders of its availability twice monthly. Partners received an initial health information group session together, after which intervention couples received a further single-sex group session and four couples counselling sessions designed to improve and promote relationship quality. Partners were separately, but simultaneously, interviewed face-to-face in isiZulu language by a gender-matched interviewer at four timepoints: baseline and 3, 6 and 9 months, with individual reports linked for analysis using a couple identifier. Responses were recorded using mobile phone data capture. Demographic and socio-economic information was collected at baseline only. Sexual, health and relationship behaviours and markers of relationship quality were collected at all timepoints.

\section{Ethics}

Ethical approval for the primary study was obtained through the Committee on Human Research of the University of California, San Francisco, the Research Ethics Committee of the Human Sciences Research Council in South Africa, and London School of Hygiene and Tropical Medicine, UK. The study protocol is available at http://www.clinicaltrials.gov. Additional detail concerning intervention and study procedures have been published previously [41]. Ethical approval for this secondary data analysis was obtained from the Ethics
Committee of the Faculty of Social, Human and Mathematical Sciences, University of Southampton.

\section{Independent Variable}

Couples' perceived access to peer support was the independent variable of interest and, for this analysis, was conceptualised by self-rated strength of agreement with two statements describing friendships outside of the primary partnership. The statements were adapted from a standardised scale measuring sense of autonomy within the relationship [42], and translated into isiZulu:

- 'I have a supportive group of friends separate from my partner' ('friends' score);

- 'I have a close friend other than my partner' ('close friend' score).

Agreement with each statement was separately scored on a nine-point scale, and scores were summed to generate a composite 'peer support' (PS) score per partner (maximum range 2 to 18 points). Within-couple mean scores were used as couple-level average (range 2 to 18 points) and female scores were subtracted from male scores to calculate couplelevel difference (range -16 to +16 points), the latter considered a potential confounder.

\section{Outcome Variable}

Although a binary outcome for CHTC uptake during followup was used for some analyses, time to uptake was also considered using discrete-time survival models. Couples were considered at risk from date of randomisation, and censored if they had not taken up CHTC by 9 months' follow-up.

\section{Adjustment Variables}

Recognising the possibility that the selected statements might also describe individuals engaging in outside relationships or activities as means of coping with an unsatisfactory primary partnership, measures of relationship satisfaction [43] and intimacy [42] were analysed as potential confounders. Each measure was adapted from a standardised scale: the former indicated by self-rating one interview item ('In general, how satisfied are you with your relationship?') on a six-point numeric scale [43]; the latter a composite measure using five interview items (for example, 'I spend as much time with my partner as possible'), each on a ninepoint numeric scale [42]. In each case couple-level average and difference scores were calculated and converted to categorical variables for analysis. Categorisations were derived following the same method as couple PS scores, outlined below. 
As around $90 \%$ of study couples were unmarried relationship status was categorised by cohabitation, which was assumed if reported by at least one partner; $94 \%$ of withincouple reports were concordant; five couples without valid report were excluded from all analyses. Within-couple age difference, partnership length, employment status, receipt of state grant (including child support grants, disability grants and workman's compensation), educational attainment (completion of secondary education, also called matric) and religion were also considered. Most variables were categorised using couple-level definitions, although male and female religion, for which around $65 \%$ of within-couple reports were discordant, were taken separately. Furthermore, as over $90 \%$ of within-couple partnership length reports differed by less than 1 year, the male report was used to represent the couple. Finally, owing to the significant intervention effect [38] all models were adjusted for trial arm; models with one additional explanatory variable are henceforth described as bivariable.

\section{Analysis}

Exploratory random effects modelling was used to assess change in couple average PS score across the four survey timepoints (results not shown). As no statistically significant change was detected, all subsequent modelling treated scores and covariates as time-invariant and fixed at baseline.

Between-group comparisons on the dichotomised outcome used the chi-squared test for categorised characteristics of the study cohort, and the Mann-Whitney test for non-normally distributed male, female and couple PS score variables. All tests used reports at baseline.

Couple average, couple difference and individual partner PS scores were analysed as categorical variables rather than assuming a linear relationship with the outcome, with exploratory analysis used to determine the most appropriate categorisations. Histograms representing the distribution of each continuous variable were created, and two-way tables between categorised variables and the outcome were used to see how the proportion meeting the outcome varied across categories. Categories were specified in line with natural groupings suggested by the histogram, with an effort to balance sample size across categories, ensure parsimony in the final models and avoid introducing heterogeneity in the relationship with the outcome within categories. Binary categorisations were used for male (at $\geq 8$ points) and female (at $\geq 10$ points) partner scores, whereas four categories (at $\leq 4,5$ to 6,7 to 10 and $\geq 11$ points) were required for couple average and five categories (at $\leq-7,-6$ to $-2,-1$ to $+1,+2$ to +6 and $\geq+7$ ) for couple difference.

To compare the relative probability of the outcome across couple average score categories, survival estimates at nine months' follow-up were calculated, using the log-rank test for significant differences [44]. Modelling time to outcome (in days) using a Cox proportional hazards specification was ruled out owing to failure to satisfy the proportional hazards assumption. Instead, discrete-time survival models (taking month as the unit of time) were used, expressing results as odds ratios. As the majority of events occurred early in the follow-up period the final four follow-up months were grouped into a single time unit.

Variables significant at the $10 \%$ level in bivariable discrete-time survival models were introduced to a multivariable model in descending order of significance, iteratively assessed using the likelihood ratio test and retained in the final model if significant at the 5\% level. Lastly, to assess the effect of male and female partner PS scores on CHTC uptake, a further model was created replacing couple average and difference PS scores with both individual partner variables.

All statistical analyses used Stata SE Version 14.0 (StataCorp, College Station, TX, USA).

\section{Results}

Of 327 couples, 88 (26.9\%) took up CHTC by nine months' follow-up. Unadjusted analysis indicated statistically significant differences by outcome for male PS score $\left(\chi^{2}=5.40, \mathrm{df}=1, \mathrm{p}=0.020\right)$, female PS score $\left(\chi^{2}=4.00\right.$, $\mathrm{df}=1, \mathrm{p}=0.045)$, couple average PS score $\left(\chi^{2}=10.87\right.$, $\mathrm{df}=3, \mathrm{p}=0.012)$, cohabitation $\left(\chi^{2}=9.70, \mathrm{df}=1, \mathrm{p}=0.002\right)$ and female-reported religion $\left(\chi^{2}=8.35, \mathrm{df}=3, \mathrm{p}=0.039\right)$ (Table 1). Couples with CHTC uptake also had lower couple average (significant; $\mathrm{z}=2.18, \mathrm{p}=0.030$ ) and male partner (borderline; $\mathrm{z}=1.92, \mathrm{p}=0.054$ ) PS scores, and significantly lower couple average $(\mathrm{z}=2.78, \mathrm{p}=0.005)$ and male partner $(\mathrm{z}=2.39, \mathrm{p}=0.017)$ scores on the 'friends' component of the overall PS score (Table 2). Furthermore, time to uptake varied over different levels of couple average PS score (logrank; $\chi^{2}=11.81, \mathrm{df}=3, \mathrm{p}=0.008$ ), with survival estimates indicating a higher likelihood and shorter time to uptake by nine months follow-up for couples with lower scores (Table 3).

An unadjusted bivariable discrete-time survival model indicated a significant association between couple average PS score and uptake $\left(\chi^{2}=13.06, \mathrm{df}=3, \mathrm{p}=0.005\right)$, with uptake less likely amongst couples with average $\geq 7$ points (OR $0.34,95 \%$ CI $0.18,0.68$ [7-10 points]; OR $0.53,95 \%$ CI $0.28,0.99$ [ $\geq 11$ points]) compared to the reference category of $\leq 4$ points (Table 4 ). In the final multivariable model adjusting for cohabitation, female-reported religion and couple difference PS score, the negative association between couple average PS score and uptake remained (AOR 0.59, 95\% CI 0.25, 1.42 [7-10 points]; AOR 0.88, 95\% CI 0.36, 
Table 1 Baseline characteristics of couples included in analysis

\begin{tabular}{|c|c|c|c|c|c|c|c|}
\hline \multirow[t]{2}{*}{ Characteristic } & \multicolumn{2}{|c|}{$\begin{array}{l}\text { No CHTC uptake } \\
\mathrm{n}=239(73.1 \%)\end{array}$} & \multicolumn{2}{|c|}{$\begin{array}{l}\text { CHTC uptake } \\
\mathrm{n}=88(26.9 \%)\end{array}$} & \multirow[t]{2}{*}{ Test statistic ${ }^{\mathrm{a}}$} & \multirow[t]{2}{*}{$\mathrm{df}$} & \multirow[t]{2}{*}{ p-value } \\
\hline & $\mathrm{n}$ & $\%$ (column) & $\mathrm{n}$ & $\%$ (column) & & & \\
\hline \multicolumn{8}{|l|}{ Male peer support score } \\
\hline 7 points or less & 96 & 40.2 & 48 & 54.6 & 5.40 & 1 & $0.020^{\mathrm{b}}$ \\
\hline 8 points or more & 143 & 59.8 & 40 & 45.5 & & & \\
\hline \multicolumn{8}{|l|}{ Female peer support score } \\
\hline 9 points or less & 128 & 53.6 & 58 & 65.9 & 4.00 & 1 & $0.045^{\mathrm{b}}$ \\
\hline 10 points or more & 111 & 46.4 & 30 & 34.1 & & & \\
\hline \multicolumn{8}{|l|}{ Couple average peer support score } \\
\hline 4 points or less & 50 & 20.9 & 26 & 29.6 & 10.87 & 3 & $0.012^{\mathrm{b}}$ \\
\hline $5-6$ points & 41 & 17.2 & 25 & 28.4 & & & \\
\hline $7-10$ points & 72 & 30.1 & 16 & 18.2 & & & \\
\hline 11 points or more & 76 & 31.8 & 21 & 23.9 & & & \\
\hline \multicolumn{8}{|l|}{ Couple difference peer support score } \\
\hline-7 points or more (female higher) & 49 & 20.5 & 18 & 20.5 & 2.71 & 4 & 0.607 \\
\hline-6 to -2 points & 40 & 16.7 & 15 & 17.1 & & & \\
\hline-1 to +1 points & 62 & 25.9 & 28 & 31.8 & & & \\
\hline+2 to +6 points & 41 & 17.2 & 16 & 18.2 & & & \\
\hline+7 points or more (male higher) & 47 & 19.7 & 11 & 12.5 & & & \\
\hline \multicolumn{8}{|l|}{ Couple average satisfaction score } \\
\hline 5 points or less & 37 & 15.5 & 8 & 9.1 & 2.21 & 1 & 0.137 \\
\hline 6 points & 202 & 84.5 & 80 & 90.9 & & & \\
\hline \multicolumn{8}{|l|}{ Couple difference satisfaction score } \\
\hline 0 points or less (equal, or female higher) & 103 & 43.1 & 35 & 39.8 & 0.29 & 1 & 0.589 \\
\hline+1 point or more (male higher) & 136 & 56.9 & 53 & 60.2 & & & \\
\hline \multicolumn{8}{|l|}{ Couple average intimacy score } \\
\hline 39 points or less & 62 & 25.9 & 19 & 21.6 & 0.68 & 2 & 0.713 \\
\hline $40-42$ points & 124 & 51.9 & 49 & 55.7 & & & \\
\hline 43 points or more & 53 & 22.2 & 20 & 22.7 & & & \\
\hline \multicolumn{8}{|l|}{ Couple difference intimacy score } \\
\hline-3 points or more (female higher) & 76 & 31.8 & 20 & 22.7 & 6.21 & 2 & $0.045^{\mathrm{b}}$ \\
\hline-2 to +2 points & 60 & 25.1 & 34 & 38.6 & & & \\
\hline+3 points or more (male higher) & 103 & 43.1 & 34 & 38.6 & & & \\
\hline \multicolumn{8}{|l|}{ Couple age difference (male minus female) } \\
\hline-2 years or more (female older) & 36 & 15.1 & 8 & 9.1 & 6.19 & 3 & 0.103 \\
\hline-1 to +1 year & 76 & 31.8 & 23 & 26.1 & & & \\
\hline+2 to +5 years & 75 & 31.4 & 40 & 45.5 & & & \\
\hline+6 years or more (male older) & 52 & 21.8 & 17 & 19.3 & & & \\
\hline \multicolumn{8}{|l|}{ Partnership length } \\
\hline Less than 2 years & 40 & 16.7 & 16 & 18.2 & 5.45 & 3 & 0.141 \\
\hline 2 to 4 years & 119 & 49.8 & 33 & 37.5 & & & \\
\hline 5 to 9 years & 51 & 21.3 & 21 & 23.9 & & & \\
\hline 10 years or more & 29 & 12.1 & 18 & 20.5 & & & \\
\hline \multicolumn{8}{|l|}{ Cohabitation } \\
\hline No & 194 & 81.2 & 57 & 64.8 & 9.70 & 1 & $0.002^{\mathrm{b}}$ \\
\hline Yes & 45 & 18.8 & 31 & 35.2 & & & \\
\hline \multicolumn{8}{|l|}{ Employment status } \\
\hline Male unemployed & 148 & 61.9 & 61 & 69.3 & 1.56 & 2 & 0.458 \\
\hline Only male employed & 69 & 28.9 & 21 & 23.9 & & & \\
\hline Both employed & 22 & 9.2 & 6 & 6.8 & & & \\
\hline
\end{tabular}


Table 1 (continued)

\begin{tabular}{|c|c|c|c|c|c|c|c|}
\hline \multirow[t]{2}{*}{ Characteristic } & \multicolumn{2}{|c|}{$\begin{array}{l}\text { No CHTC uptake } \\
\mathrm{n}=239(73.1 \%)\end{array}$} & \multicolumn{2}{|c|}{$\begin{array}{l}\text { CHTC uptake } \\
\mathrm{n}=88(26.9 \%)\end{array}$} & \multirow[t]{2}{*}{ Test statistic ${ }^{\mathrm{a}}$} & \multirow[t]{2}{*}{$\mathrm{df}$} & \multirow[t]{2}{*}{ p-value } \\
\hline & $\mathrm{n}$ & $\%$ (column) & $\mathrm{n}$ & $\%$ (column) & & & \\
\hline \multicolumn{8}{|l|}{ Receipt of state employment grant } \\
\hline Neither partner receives grant & 117 & 49.0 & 37 & 42.1 & 1.23 & 1 & 0.267 \\
\hline At least one partner receives grant & 122 & 51.1 & 51 & 58.0 & & & \\
\hline \multicolumn{8}{|l|}{ Educational attainment } \\
\hline Both have matric or higher & 63 & 26.4 & 17 & 19.3 & 4.85 & 3 & 0.184 \\
\hline Only male has matric or higher & 47 & 19.7 & 14 & 15.9 & & & \\
\hline Only female has matric or higher & 51 & 21.3 & 17 & 19.3 & & & \\
\hline Both have incomplete secondary or lower & 78 & 32.6 & 40 & 45.5 & & & \\
\hline \multicolumn{8}{|l|}{ Religion (female reported) } \\
\hline None & 18 & 7.5 & 16 & 18.2 & 8.35 & 3 & $0.039^{\mathrm{b}}$ \\
\hline Christian & 135 & 56.5 & 42 & 47.7 & & & \\
\hline Zionist & 61 & 25.5 & 23 & 26.1 & & & \\
\hline Other & 25 & 10.5 & 7 & 8.0 & & & \\
\hline \multicolumn{8}{|l|}{ Religion (male reported) } \\
\hline None & 86 & 36.0 & 40 & 45.5 & 6.37 & 3 & $0.095^{\mathrm{c}}$ \\
\hline Christian & 98 & 41.0 & 27 & 30.7 & & & \\
\hline Zionist & 38 & 15.9 & 10 & 11.4 & & & \\
\hline Other & 17 & 7.1 & 11 & 12.5 & & & \\
\hline
\end{tabular}

${ }^{\mathrm{a}} \mathrm{Chi}$-squared test statistic

${ }^{\mathrm{b}}$ Significant at $\mathrm{p}<0.05$ level

${ }^{\mathrm{c}}$ Significant at $\mathrm{p}<0.10$ level

Table 2 Distribution of male, female and couple peer support and component scores at baseline, by outcome

\begin{tabular}{lllll}
\hline Variable & $\begin{array}{l}\text { No CHTC uptake } \\
\mathrm{n}=239(73.1 \%) \\
\text { Median (IQR) }\end{array}$ & $\begin{array}{l}\text { CHTC uptake } \\
\mathrm{n}=88(26.9 \%) \\
\text { Median (IQR) }\end{array}$ & Test statistic & p-value \\
\hline Peer support score & & & \\
Male & $9.0(2.0,13.0)$ & $5.0(2.0,11.0)$ & 1.92 & $0.054^{\mathrm{c}}$ \\
Female & $9.0(3.0,16.0)$ & $8.5(3.0,10.0)$ & 1.02 & 0.310 \\
Couple average & $9.0(5.5,12.0)$ & $6.5(3.0,10.5)$ & 2.18 & $0.030^{\mathrm{b}}$ \\
Couple difference (male minus female) & $0.0(-6.0,5.0)$ & $0.0(-4.5,3.0)$ & 0.42 & 0.672 \\
'Friends' score & & & & \\
Male & $5.0(1.0,7.0)$ & $2.0(1.0,7.0)$ & 2.39 & $0.017^{\mathrm{b}}$ \\
Female & $2.0(1.0,8.0)$ & $2.0(1.0,8.0)$ & 1.44 & 0.149 \\
Couple average & $4.5(1.5,6.5)$ & $3.5(1.5,4.75)$ & 2.78 & $0.005^{\mathrm{b}}$ \\
Couple difference (male minus female) & $0.0(-1.0,3.0)$ & $0.0(-1.0,1.0)$ & 0.39 & 0.698 \\
'Close friend' score & & & & \\
Male & $2.0(1.0,7.0)$ & $2.0(1.0,7.0)$ & 0.66 & 0.510 \\
Female & $2.0(1.0,8.0)$ & $2.0(1.0,8.0)$ & 0.14 & 0.893 \\
Couple average & $4.5(1.5,5.5)$ & $4.25(1.5,5.0)$ & 0.56 & 0.574 \\
Couple difference (male minus female) & $0.0(-2.0,1.0)$ & $0.0(-1.0,1.0)$ & 0.37 & 0.710 \\
\hline
\end{tabular}

${ }^{a}$ Mann-Whitney test statistic

${ }^{\mathrm{b}}$ Significant at $\mathrm{p}<0.05$ level

${ }^{\mathrm{c}}$ Significant at $\mathrm{p}<0.10$ level 
Table 3 Survival estimates and log-rank test results by couple average peer support score

\begin{tabular}{lll}
\hline Measure & Category & Estimate $(95 \% \mathrm{CI})$ \\
\hline Probability of outcome by nine months' follow-up & 4 points or less & $0.65(0.53,0.75)$ \\
& $5-6$ points & $0.61(0.48,0.72)$ \\
& $7-10$ points & $0.80(0.69,0.87)$ \\
& 11 points or more & $0.78(0.68,0.85)$ \\
& Test statistic & 11.81 \\
& df & 3 \\
& p-value & 0.008
\end{tabular}

${ }^{\mathrm{a}}$ Log-rank test statistic

Table 4 Bivariable and multivariable associations between couple average peer support score and CHTC uptake

\begin{tabular}{|c|c|c|c|c|c|c|c|c|c|}
\hline \multirow[t]{2}{*}{ Variable } & \multirow{2}{*}{$\begin{array}{l}\mathrm{n} \\
(\% \text { with outcome })\end{array}$} & \multicolumn{4}{|l|}{ Bivariable Model } & \multicolumn{4}{|l|}{ Multivariable Model } \\
\hline & & OR $(95 \% \mathrm{CI})$ & Test statistic ${ }^{\mathrm{a}}$ & df & $\mathrm{p}$-value & $\begin{array}{l}\text { Adjusted OR (95\% } \\
\text { CI) }\end{array}$ & Test statistic ${ }^{a}$ & $\mathrm{df}$ & $\mathrm{p}$-value \\
\hline \multicolumn{10}{|l|}{$\begin{array}{l}\text { Couple average peer } \\
\text { support score }\end{array}$} \\
\hline 4 points or less & $76(34)$ & Reference & 13.06 & 3 & $0.005^{\mathrm{c}}$ & Reference & 6.55 & 3 & $0.088^{\mathrm{d}}$ \\
\hline $5-6$ points & $66(38)$ & $0.88(0.48,1.63)$ & & & & $1.44(0.55,3.75)$ & & & \\
\hline $7-10$ points & 88 (18) & $0.34(0.18,0.68)$ & & & & $0.59(0.25,1.42)$ & & & \\
\hline 11 points or more & $97(22)$ & $0.53(0.28,0.99)$ & & & & $0.88(0.36,2.10)$ & & & \\
\hline \multicolumn{10}{|l|}{$\begin{array}{l}\text { Couple difference } \\
\text { peer support score }\end{array}$} \\
\hline $\begin{array}{l}-7 \text { points or more } \\
\text { (female higher) }\end{array}$ & $67(27)$ & $0.73(0.39,1.39)$ & 3.28 & 4 & 0.512 & $0.81(0.32,2.07)$ & 1.14 & 4 & 0.887 \\
\hline-6 to -2 points & $55(27)$ & $0.74(0.38,1.46)$ & & & & $0.93(0.39,2.18)$ & & & \\
\hline-1 to +1 point & $90(31)$ & Reference & & & & Reference & & & \\
\hline+2 to +6 points & $57(28)$ & $0.83(0.43,1.62)$ & & & & $1.08(0.44,2.68)$ & & & \\
\hline $\begin{array}{l}+7 \text { points or more } \\
\text { (male higher) }\end{array}$ & $58(19)$ & $0.52(0.25,1.10)$ & & & & $0.70(0.26,1.89)$ & & & \\
\hline \multicolumn{10}{|l|}{ Cohabitation } \\
\hline No & $251(23)$ & Reference & 13.93 & 1 & $<0.001^{\mathrm{b}}$ & Reference & 7.98 & 1 & $0.005^{\mathrm{c}}$ \\
\hline Yes & $76(41)$ & $2.64(1.61,4.32)$ & & & & $2.48(1.42,4.34)$ & & & \\
\hline \multicolumn{10}{|l|}{$\begin{array}{l}\text { Religion (female } \\
\text { reported) }\end{array}$} \\
\hline Christian & $177(24)$ & Reference & 7.50 & 3 & $0.057^{\mathrm{d}}$ & Reference & 8.79 & 3 & $0.032^{\mathrm{c}}$ \\
\hline None & $34(47)$ & $2.46(1.28,4.71)$ & & & & $2.56(1.29,5.07)$ & & & \\
\hline Zionist & $84(27)$ & $1.08(0.63,1.87)$ & & & & $0.97(0.55,1.72)$ & & & \\
\hline Other & $32(22)$ & $0.83(0.36,1.94)$ & & & & $0.67(0.28,1.63)$ & & & \\
\hline
\end{tabular}

$\mathrm{N}=327$ couples (all models)

All models were also adjusted for trial arm

${ }^{\text {a }}$ Test statistic from likelihood ratio test of additional explanatory variable

${ }^{\mathrm{b}}$ Significant at $\mathrm{p}<0.001$ level

${ }^{c}$ Significant at $\mathrm{p}<0.05$ level

${ }^{d}$ Significant at $p<0.10$ level

$2.10[\geq 11$ points $])$, but was no longer significant $\left(\chi^{2}=6.55\right.$, $\mathrm{df}=3, \mathrm{p}=0.088$ ).

When separately included in bivariable models adjusting for trial arm, male $\left(\chi^{2}=5.74, \mathrm{df}=1, \mathrm{p}=0.017\right)$ and female $\left(\chi^{2}=4.55, \mathrm{df}=1, \mathrm{p}=0.033\right)$ PS score were each significantly associated with uptake (Table 5). Although male and female variables used different category boundaries their estimated effect sizes were similar (male OR 0.58, $95 \%$ CI $0.37,0.91$ [ $\geq 8$ points]; female OR $0.60,95 \%$ CI $0.38,0.97$ [ $\geq 10$ points]), and remained comparable (but no 
Table 5 Bivariable and multivariable associations between partner-level peer support score and CHTC uptake

\begin{tabular}{|c|c|c|c|c|c|c|c|c|c|}
\hline \multirow[t]{2}{*}{ Variable } & \multirow{2}{*}{$\begin{array}{l}\mathrm{n} \\
(\% \text { with outcome })\end{array}$} & \multicolumn{4}{|l|}{ Bivariable Model } & \multicolumn{4}{|l|}{ Multivariable Model } \\
\hline & & OR $(95 \% \mathrm{CI})$ & Test statistic ${ }^{a}$ & df & p-value & $\begin{array}{l}\text { Adjusted OR (95\% } \\
\text { CI) }\end{array}$ & Test statistic ${ }^{a}$ & $\mathrm{df}$ & $\mathrm{p}$-value \\
\hline \multicolumn{10}{|l|}{$\begin{array}{l}\text { Male peer support } \\
\text { score }\end{array}$} \\
\hline 7 points or less & $144(33)$ & Reference & 5.74 & 1 & $0.017^{\mathrm{c}}$ & Reference & 1.61 & 1 & 0.205 \\
\hline 8 points or more & $183(22)$ & $0.58(0.37,0.91)$ & & & & $0.73(0.45,1.18)$ & & & \\
\hline \multicolumn{10}{|l|}{$\begin{array}{l}\text { Female peer support } \\
\text { score }\end{array}$} \\
\hline 9 points or less & $186(31)$ & Reference & 4.55 & 1 & $0.033^{\mathrm{c}}$ & Reference & 1.54 & 1 & 0.215 \\
\hline 10 points or more & $141(21)$ & $0.60(0.38,0.97)$ & & & & $0.73(0.44,1.20)$ & & & \\
\hline \multicolumn{10}{|l|}{ Cohabitation } \\
\hline No & $251(23)$ & Reference & 13.93 & 1 & $<0.001^{\mathrm{b}}$ & Reference & 8.68 & 1 & $0.003^{\mathrm{c}}$ \\
\hline Yes & $76(41)$ & $2.64(1.61,4.32)$ & & & & $2.39(1.40,4.08)$ & & & \\
\hline \multicolumn{10}{|l|}{$\begin{array}{l}\text { Religion (female } \\
\text { reported) }\end{array}$} \\
\hline Christian & $177(24)$ & Reference & 7.50 & 3 & $0.057^{\mathrm{d}}$ & Reference & 8.31 & 3 & $0.040^{\mathrm{c}}$ \\
\hline None & $34(47)$ & $2.46(1.28,4.71)$ & & & & $2.48(1.28,4.82)$ & & & \\
\hline Zionist & $84(27)$ & $1.08(0.63,1.87)$ & & & & $0.91(0.52,1.60)$ & & & \\
\hline Other & $32(22)$ & $0.83(0.36,1.94)$ & & & & $0.77(0.32,1.82)$ & & & \\
\hline
\end{tabular}

$\mathrm{N}=327$ couples (all models)

All models were also adjusted for trial arm

${ }^{a}$ Test statistic from likelihood ratio test of additional explanatory variable

${ }^{\mathrm{b}}$ Significant at $\mathrm{p}<0.001$ level

${ }^{\mathrm{c}}$ Significant at $\mathrm{p}<0.05$ level

${ }^{\mathrm{d}}$ Significant at $\mathrm{p}<0.10$ level

longer significant) when included together in a multivariable model (male $\chi^{2}=1.61, \mathrm{df}=1, \mathrm{p}=0.205$, AOR $0.73,95 \% \mathrm{CI}$ $0.45,1.18$ [ $\geq 8$ points]; female $\chi^{2}=1.54, \mathrm{df}=1, \mathrm{p}=0.215$, AOR $0.73,95 \%$ CI $0.44,1.20$ [ $\geq 10$ points]).

\section{Discussion}

We aimed to evaluate the association between couples' perceived access to peer support and CHTC uptake in a high HIV prevalence area of South Africa. Many studies have described a social influence upon individuals' uptake of HIV testing in SSA, with support from family, friends and peers frequently reported as an enabler and fears of stigmatisation and social exclusion as barriers [13]. Our findings point to a role in relation to couples contemplating testing together, suggesting that CHTC uptake by 9 months' follow-up was less likely amongst couples with greater perceived access to peer support, conceptualised in terms of self-reported agreement with statements describing friendships outside of the primary partnership. In line with demographic trends in South Africa [45], most couples in our study cohort were unmarried and non-cohabiting, and cohabitation was a significant predictor of CHTC uptake in our bivariable and multivariable models. Previous qualitative research has suggested that non-cohabitation could contribute to worse intimacy and communication between partners in this setting [15], which may lead to relationships outside of the primary partnership becoming a more important source of personal support, giving greater prominence to normative attitudes around HIV testing and other health behaviours as expressed by friends and peers. Critically, and in contrast to testing programmes offered to individuals, the decision to participate in couples testing may result in knowledge that could be damaging to the relationship [7,46], and must be taken jointly and accepted by both partners. Although CHTC has been proposed as a means of expanding testing, and reaching men in particular [47], men have tended to be less supportive of testing together than women $[46,48,49]$. The social context has been recognised as a structural influence on couples' health and HIV risk behaviours [18] and, by acting independently and differentially upon each partner, could further constrain interactions and communication and exacerbate differences within the relationship, limiting capacity for behaviour change under the interdependence model [25].

The precise mechanism of influence is unclear owing to the composition of our independent variable of interest. 
Prioritisation of the couple average PS score could mask some aspects of individual partners' contrasting exposures, although the finding that couple difference score was not a significant predictor in bivariable or multivariable models suggests that the magnitude of difference between partners was less important than the level of their average score. In addition, the meaning of the association is likely to be qualified by participants' interpretation of the statements describing friendships outside of the primary partnership. For those assigning greater value to closer relationships, reported scores may describe exposure to the influence of a small number of trusted friends, whereas for those engaged with larger peer groups they may be more reflective of normative beliefs and attitudes circulating amongst peers. Nonetheless, our findings do suggest differing mechanisms of influence between the sexes. First, unadjusted comparisons by outcome indicated higher 'friends' and PS scores amongst males in couples without CHTC uptake, against little difference in female scores on any measure. Second, while male and female partner scores were significant predictors in separate bivariable models, binary categorisation at different boundaries indicated that males required a lower score to reach a similar couple-level effect to females. This may suggest that women were more influenced by primary relationship quality, while men tended to value peer group relationships more highly, and were more strongly influenced by them. This finding may align with previous research linking risk behaviours and attitudes to health-seeking to characteristics of male peer groups [29, 31, 50], and associating social norms more strongly with men's, rather than women's, willingness to test [51].

Couples-focused studies and interventions may be strengthened by considering the wider social context and addressing sources of support and influence outside of the primary partnership. Future research could explore the applicability of integrating existing peer support or social network measurement instruments into baseline couple assessments, or developing instruments to capture couplespecific social information, such as shared social contacts with potential to exert influence on both partners. An understanding of participating couples' social context at recruitment could help to inform intervention design; they might, for example, seek to build positive social support structures from existing peer groups, or incorporate education or counselling components to help partners prioritise their relationship and build resilience in the face of harmful social influences [52]. Notably, some men are able to recast their conception of masculinity in terms emphasising social and familial responsibility and more compatible with healthseeking and preventive behaviours [28,53], for example.

\section{Strengths and Limitations}

This secondary analysis used data from a prospective study evaluating a couples-focused behavioural intervention; a key strength, therefore, was use of a dataset allowing for longitudinal analysis at individual partner and couple levels. Multivariable effect sizes were similar to those present in bivariable models but were no longer statistically significant; the cohort was large for a study of this nature, yet increased sample size would have improved the precision of estimates and may have confirmed multivariable findings as significant. Having defined our independent variable of interest using proxy measures capturing only one specific kind of social relationship our analysis can only provide a limited picture of social influence on this couples-focused outcome, but was designed as initial exploration of an area that, to our knowledge, has not yet been substantively addressed. A social network analysis examining couples' overall network composition including the number, closeness and significance of relationships with friends, family members and other acquaintances, and individual and shared connections, may provide further clarity and nuance around the observed association. Self-reported PS scores may also be subject to social desirability bias if overstated to present a more favourable self-image or understated to emphasise dissatisfaction. Finally, voluntary participation in the primary study may have introduced self-selection bias if it attracted couples most willing to participate in such interventions, with potential to limit generalisability.

\section{Conclusions}

Interventions designed to expand HIV testing and facilitate mutual disclosure amongst couples could be impactful in SSA, given the inherently dyadic dimension to transmission in this setting, but our findings suggest that, for some couples, social influences may create a structural barrier to uptake. The proximity and ubiquity of relationships and interactions within the lived social environment may mean that they resonate more strongly with individuals and couples in their capacity to influence, or discourage, behaviour change, presenting an important counterpoint to HIV prevention programmes. Accounting for that environment in the design of couples-focused interventions may increase their success, and could offer further opportunity to improve the wider appeal and acceptability of HIV prevention behaviours, contributing to a more healthful social context for all.

Author Contributions MJJ carried out this secondary data analysis and wrote the manuscript. LAD obtained funding for, designed and directed the implementation of the primary study, and provided clinical guidance for this secondary data analysis. NM contributed to the design and participated in the implementation of the primary study, and provided statistical and methodological oversight and guidance for this secondary data analysis. VH, MOJ and KF contributed to the 
design and participated in the implementation of the primary study. TN led the implementation of the primary study and supervised the study staff. HVR contributed to the design and directed the implementation of the primary study. All authors commented on drafts, and have read and approved the final manuscript.

Funding MJJ was funded by the UK National Institute for Health Research Applied Research Collaboration Wessex at University Hospital Southampton NHS Foundation Trust. The primary study and other authors were funded by the National Institutes of Health (R01 MH086346) to Principal Investigator LAD. NM was also supported by a Wellcome Trust fellowship (grant number WT083495MA) and is a recipient of an NIHR Research Professorship award (Ref: RP2017-08-ST2-008). VH was also supported by the UK Economic and Social Research Council (ES/J021202/1).

Data Availability The datasets generated during and/or analysed during the current study are available from the Principal Investigator (Lynae Darbes) on reasonable request, and also available upon request from the Human Sciences Research Council, South Africa.

\section{Declarations}

Conflict of interest The authors declare that they have no conflict of interest.

Ethical Approval Ethical approval for the primary study was obtained through the Committee on Human Research of the University of California, San Francisco, the Research Ethics Committee of the Human Sciences Research Council in South Africa, and London School of Hygiene and Tropical Medicine, UK. The study protocol is available at http://www.clinicaltrials.gov. Additional detail concerning intervention and study procedures have been published previously. Ethical approval for this secondary data analysis was obtained from the Ethics Committee of the Faculty of Social, Human and Mathematical Sciences, University of Southampton.

Informed Consent Informed consent was obtained from all individual participants included in the study.

Open Access This article is licensed under a Creative Commons Attribution 4.0 International License, which permits use, sharing, adaptation, distribution and reproduction in any medium or format, as long as you give appropriate credit to the original author(s) and the source, provide a link to the Creative Commons licence, and indicate if changes were made. The images or other third party material in this article are included in the article's Creative Commons licence, unless indicated otherwise in a credit line to the material. If material is not included in the article's Creative Commons licence and your intended use is not permitted by statutory regulation or exceeds the permitted use, you will need to obtain permission directly from the copyright holder. To view a copy of this licence, visit http://creativecommons.org/licenses/by/4.0/.

\section{References}

1. Chemaitelly H, Awad SF, Shelton JD, Abu-Raddad LJ. Sources of HIV incidence among stable couples in sub-Saharan Africa. J Int AIDS Soc. 2014; 17:18765.

2. Dunkle KL, Stephenson R, Karita E, Chomba E, Kayitenkore $\mathrm{K}$, Vwalika C, et al. New heterosexually transmitted HIV infections in married or cohabiting couples in urban Zambia and Rwanda: an analysis of survey and clinical data. Lancet. 2008:371(9631):2183-91.

3. Chemaitelly H, Cremin I, Shelton J, Hallett TB, Abu-Raddad LJ. Distinct HIV discordancy patterns by epidemic size in stable sexual partnerships in sub-Saharan Africa. Sex Transm Infect. 2012;88(1):51-7.

4. de Walque D. Sero-discordant couples in five African countries: implications for prevention strategies. Popul Dev Rev. 2007;33(3):501-23.

5. Lasry A, Sansom SL, Wolitski RJ, Green TA, Borkowf CB, Patel $\mathrm{P}$, et al. HIV sexual transmission risk among serodiscordant couples: assessing the effects of combining prevention strategies. AIDS. 2014;28(10):1521-9.

6. Reynolds SJ, Makumbi F, Nakigozi G, Kagaayi J, Gray RH, Wawer M, et al. HIV-1 transmission among HIV-1 discordant couples before and after the introduction of antiretroviral therapy. AIDS. 2011;25(4):473-7.

7. Tabana H, Doherty T, Rubenson B, Jackson D, Ekstrom AM, Thorson A. "Testing together challenges the relationship": Consequences of HIV testing as a couple in a high HIV prevalence setting in rural South Africa. PLoS ONE. 2013;8(6):e66390.

8. Bunnell R, Opio A, Musinguzi J, Kirungi W, Ekwaru P, Mishra V, et al. HIV transmission risk behavior among HIV-infected adults in Uganda: results of a nationally representative survey. AIDS. 2008;22(5):617-24.

9. Rosenberg NE, Pettifor AE, De Bruyn G, Westreich D, DelanyMoretlwe S, Behets F, et al. HIV testing and counseling leads to immediate consistent condom use among South African stable HIV-discordant couples. J Acquir Immune Defic Syndr. 2013;62(2):226-33.

10. Cherutich P, Kaiser R, Galbraith J, Williamson J, Shiraishi RW, Ngare C, et al. Lack of knowledge of HIV status a major barrier to HIV prevention, care and treatment efforts in Kenya: results from a nationally representative study. PLoS ONE. 2012;7(5):e36797.

11. Dokubo EK, Shiraishi RW, Young PW, Neal JJ, Aberle-Grasse J, Honwana N, et al. Awareness of HIV status, prevention knowledge and condom use among people living with HIV in Mozambique. PLoS ONE. 2014;9(9):e106760.

12. Peltzer K, Matseke G, Mzolo T, Majaja M. Determinants of knowledge of HIV status in South Africa: results from a population-based HIV survey. BMC Public Health. 2009;9:174.

13. Musheke M, Ntalasha H, Gari S, McKenzie O, Bond V, MartinHilber A, et al. A systematic review of qualitative findings on factors enabling and deterring uptake of HIV testing in Sub-Saharan Africa. BMC Public Health. 2013;13:220.

14. Camlin CS, Ssemmondo E, Chamie G, El Ayadi AM, Kwarisiima D, Sang N, et al. Men "missing" from population-based HIV testing: insights from qualitative research. AIDS Care. 2016;28(Suppl 3):67-73.

15. Chikovore J, Gillespie N, McGrath N, Orne-Gliemann J, Zuma $\mathrm{T}$. Men, masculinity, and engagement with treatment as prevention in KwaZulu-Natal, South Africa. AIDS Care. 2016;28(Suppl 3):74-82.

16. Kennedy CE, Fonner VA, Armstrong KA, O'Reilly KR, Sweat MD. Increasing HIV serostatus disclosure in low and middleincome countries: a systematic review of intervention evaluations. AIDS. 2015;29(Suppl 1):S7-23.

17. McGrath N, Richter L, Newell ML. Sexual risk after HIV diagnosis: a comparison of pre-ART individuals with CD4 > 500 cells/ microl and ART-eligible individuals in a HIV treatment and care programme in rural KwaZulu-Natal, South Africa. J Int AIDS Soc. 2013;16:18048.

18. Karney BR, Hops H, Redding CA, Reis HT, Rothman AJ, Simpson JA. A framework for incorporating dyads in models of HIVprevention. AIDS Behav. 2010;14(Suppl 2):189-203. 
19. Montgomery CM, Watts C, Pool R. HIV and dyadic intervention: an interdependence and communal coping analysis. PLoS ONE. 2012;7(7):e40661.

20. Burton J, Darbes LA, Operario D. Couples-focused behavioral interventions for prevention of HIV: systematic review of the state of evidence. AIDS Behav. 2010;14(1):1-10.

21. Crepaz N, Tungol-Ashmon MV, Vosburgh HW, Baack BN, Mullins MM. Are couple-based interventions more effective than interventions delivered to individuals in promoting HIV protective behaviors? A meta-analysis. AIDS Care. 2015;27(11):1361-6.

22. Wall KM, Kilembe W, Vwalika B, Haddad LB, Lakhi S, Onwubiko U, et al. Sustained effect of couples' HIV counselling and testing on risk reduction among Zambian HIV serodiscordant couples. Sex Transm Infect. 2017;93(4):259-66.

23. World Health Organisation. Guidance on Couples HIV Testing and Counselling Including Antiretroviral Therapy for Treatment and Prevention in Serodiscordant Couples: Recommendations for a Public Health Approach 2012 http://www.who.int/hiv/pub/guide lines/9789241501972/en/.

24. Hailemariam TG, Nathan S, Seifu CN, Rawstorne P. Uptake of couples HIV testing and counselling among heterosexual couples in Sub-Saharan Africa: a systematic review and meta-analysis. AIDS Care. 2020;32(2):137-47.

25. Lewis MA, McBride CM, Pollak KI, Puleo E, Butterfield RM, Emmons KM. Understanding health behavior change among couples: an interdependence and communal coping approach. Soc Sci Med. 2006;62(6):1369-80.

26. Latkin CA, Knowlton AR. Micro-social structural approaches to HIV prevention: a social ecological perspective. AIDS Care. 2005;17(Suppl 1):S102-13.

27. Izugbara CO, Undie C, Mudege NN, Ezeh AC. Male youth and voluntary counseling and HIV-testing: the case of Malawi and Uganda. Sex Educ. 2009;9(3):243-59.

28. Skovdal M, Campbell C, Madanhire C, Mupambireyi Z, Nyamukapa C, Gregson S. Masculinity as a barrier to men's use of HIV services in Zimbabwe. Global Health. 2011;7:13.

29. Yamanis TJ, Fisher JC, Moody JW, Kajula LJ. Young men's social network characteristics and associations with sexual partnership concurrency in Tanzania. AIDS Behav. 2016;20(6):1244-55.

30. Clark S. Extra-marital sexual partnerships and male friendships in rural Malawi. Demogr Res. 2010;22:1-28.

31. Cordero CJ. When the group encourages extramarital sex: Difficulties in HIV/AIDS prevention in rural Malawi. Demogr Res. 2013;28:849-80

32. Allen S, Karita E, Chomba E, Roth DL, Telfair J, Zulu I, et al. Promotion of couples' voluntary counselling and testing for HIV through influential networks in two African capital cities. BMC Public Health. 2007;7:349

33. Wall K, Karita E, Nizam A, Bekan B, Sardar G, Casanova D, et al. Influence network effectiveness in promoting couples' HIV voluntary counseling and testing in Kigali, Rwanda. AIDS. 2012;26(2):217-27.

34. Norr KF, Norr JL, McElmurry BJ, Tlou S, Moeti MR. Impact of peer group education on HIV prevention among women in Botswana. Health Care Women Int. 2004;25(3):210-26.

35. Maman S, Mulawa MI, Balvanz P, Reyes HLM, Kilonzo MN, Yamanis TJ, et al. Results from a cluster-randomized trial to evaluate a microfinance and peer health leadership intervention to prevent HIV and intimate partner violence among social networks of Tanzanian men. PLoS ONE. 2020;15(3):e0230371.

36. Kaponda CP, Norr KF, Crittenden KS, Norr JL, McCreary LL, Kachingwe SI, et al. Outcomes of an HIV prevention peer group intervention for rural adults in Malawi. Health Educ Behav. 2011;38(2):159-70.
37. Njiru R. Social networks, gender and HIV within marriages in Kenya. Afr J AIDS Res. 2019;18(3):224-33.

38. Darbes LA, McGrath NM, Hosegood V, Johnson MO, Fritz $\mathrm{K}$, Ngubane $\mathrm{T}$, et al. Results of a couples-based randomized controlled trial aimed to increase testing for HIV. JAIDS. 2019;80(4):404-13.

39. Statistics South Africa. Census 2011: Census In Brief 2012 http:// www.statssa.gov.za/?page_id $=3955$.

40. Shisana O, Rehle T, Simbayi LC, Zuma K, Jooste S, Zungu N, et al. South African National HIV Prevalence, Incidence and Behaviour Survey, 2012. Cape Town: HSRC Press; 2014.

41. Darbes LA, van Rooyen H, Hosegood V, Ngubane T, Johnson MO, Fritz K, et al. Uthando Lwethu ('our love'): a protocol for a couples-based intervention to increase testing for HIV: a randomized controlled trial in rural KwaZulu-Natal, South Africa. Trials. 2014;15:64.

42. Kurdek LA. The deterioration of relationship quality for gay and lesbian cohabiting couples: a five-year prospective longitudinal study. Pers Relat. 1996;3:417-42.

43. Funk JL, Rogge RD. Testing the ruler with item response theory: increasing precision of measurement for relationship satisfaction with the Couples Satisfaction Index. J Fam Psychol. 2007;21(4):572-83.

44. Collett D. Modelling survival data in medical research. 3rd ed. Boca Raton: Chapman and Hall/CRC Press; 2003.

45. Hosegood V, McGrath N, Moultrie T. Dispensing with marriage: marital and partnership trends in rural KwaZulu-Natal, South Africa 2000-2006. Demogr Res. 2009;20:279-312.

46. Njau B, Watt MH, Ostermann J, Manongi R, Sikkema KJ. Perceived acceptability of home-based couples voluntary HIV counseling and testing in Northern Tanzania. AIDS Care. 2012;24(4):413-9.

47. Sharma M, Barnabas RV, Celum C. Community-based strategies to strengthen men's engagement in the HIV care cascade in subSaharan Africa. PLoS Med. 2017;14(4):e1002262.

48. Matovu JK, Wanyenze RK, Wabwire-Mangen F, Nakubulwa R, Sekamwa R, Masika A, et al. "Men are always scared to test with their partners ... it is like taking them to the Police": Motivations for and barriers to couples' HIV counselling and testing in Rakai, Uganda: a qualitative study. J Int AIDS Soc. 2014;17:19160.

49. Nannozi V, Wobudeya E, Gahagan J. Fear of an HIV positive test result: an exploration of the low uptake of couples HIV counselling and testing (CHCT) in a rural setting in Mukono district, Uganda. Glob Health Promot. 2017;24(4):33-42.

50. Mulawa M, Yamanis TJ, Hill LM, Balvanz P, Kajula LJ, Maman S. Evidence of social network influence on multiple HIV risk behaviors and normative beliefs among young Tanzanian men. Soc Sci Med. 2016;153:35-43.

51. Babalola S. Readiness for HIV testing among young people in northern Nigeria: the roles of social norm and perceived stigma. AIDS Behav. 2007;11(5):759-69.

52. Conroy AA, Ruark A, McKenna SA, Tan JY, Darbes LA, Hahn JA, et al. The unaddressed needs of alcohol-using couples on antiretroviral therapy in Malawi: formative research on multilevel interventions. AIDS Behav. 2019;24(6):1599-611.

53. Hosegood V, Richter L, Clarke L. “...I should maintain a healthy life now and not just live as i please...": men's health and fatherhood in rural South Africa. Am J Mens Health. 2016;10(6):39-50.

Publisher's Note Springer Nature remains neutral with regard to jurisdictional claims in published maps and institutional affiliations. 